\title{
Normalization in Comparative Life Cycle Assessment to Support Environmental Decision Making
}

\author{
Stefano Cucurachi, ${ }^{1}$ Thomas $P$ Seager, ${ }^{2}$ and Valentina Prado ${ }^{3}$ \\ ${ }^{1}$ Bren School of Environmental Science $\mathcal{B}$ Management, University of California, Santa Barbara, CA, USA \\ ${ }^{2}$ School of Sustainable Engineering and the Built Environment, Arizona State University, Tempe, AZ, USA \\ ${ }^{3}$ Institute of Environmental Sciences (CML), Department of Industrial Ecology, Leiden University, Leiden, \\ the Netherlands
}

\section{Introduction}

Although it is now widely accepted that the proper perspective for analysis of environmental decisions is the life cycle perspective (ISO 2006), existing practices of life cycle assessment (LCA) still lack connection to structured approaches for environmental decision making. As a consequence, comparative LCA studies typically leave decision makers to confront complex decision problems without the aid of the analytical tools necessary to make trade-offs clear.

It is only in the normalization and weighting steps of LCA (ISO 2006) that decision-analytic requirements can be met. However, formal decision techniques are " . . . it is only by providing context through normalization that data can be made meaningful for decisions. That is, better data alone is insufficient for extracting meaning." here often ignored. A typical practice of the normalization step consists of dividing characterized results of a product system by the characterized total emissions within a political or geographical boundary (e.g., European Union, United States, or global) or industry for a certain period of time (e.g., 1 year). This approach should allow understanding the magnitude of emissions that can be attributed to the production of a marginal functional unit, as compared to a reference system, and is often referred to as external normalization (Norris 2001).

Although the results are intended to be reproducible and scientifically defensible, years of experience with external normalization have revealed several obstacles to these goals. In particular, numerous studies documented the practical difficulties of obtaining, maintaining, and repairing reliable external normalization reference data sets (see Pizzol et al. [2016], among others). However, even perfect information and complete normalization references would not overcome more-profound objections to external normalization. Recent examinations reveal

(C) 2017 by Yale University

DOI: $10.1111 /$ jiec. 12549

Volume 0 , Number 0 that the practice may mask trade-offs, rather than reveal them, and bias results such that the normalization approach alone dominates comparative results, irrespective of the differences in the inventory, weighting schemes, or stakeholder objectives (Prado-Lopez et al. 2014).

The alternative approach to external normalization commonly taken in the literature is internal normalization (Norris 2001), which focuses exclusively on the relative differences among the alternatives under consideration using internal data, rather than on their assessment with respect to absolute external standards. The recognized pitfalls of the internal approach include compensation, logical traps, and magnitude insensitivity (Norris 2001).

\section{Innovation in Normalization}

Two new approaches to normalization have been proposed that partially overcome current obstacles to normalization in comparative LCA. The first is external and proposes changing the reference data set from existing emissions to target emissions levels (Bjorn and Hauschild 2015). Following this approach replaces the descriptive problem of monitoring emissions inventories with the prescriptive problem of establishing external reference levels of acceptable emissions according to scientific determination of global carrying capacity, maximum local daily pollutant loads, or sociopolitical processes reflecting subjective values. The shift from levels to targets may make bias more transparent and provide a context for identifying hotspots for a specific product system in relationship to global trends, challenges, or goals.

Alternatively, for the purposes of aggregation of results in comparative studies, the second advancement in normalization is internal. The proposed approach uses internal normalization, such as pair-wise outranking, in structured decision-analytic techniques that are suitable for environmental decision making (Prado-Lopez et al. 2014). The use of these techniques 
ameliorate past criticisms of internal normalization, enhance the congruency between normalization and weighting, and enable the inclusion of uncertainty and stakeholder values directly in the analysis.

Under current standards, normalization is optional (ISO 2006) and many studies truncate LCA at the characterization step. Given the biases in current practices, skipping the normalization step may be preferable to the methods that dominated practice at the time the International Standards Organization (ISO) codified the consensus. Nevertheless, it is only by providing context through normalization that data can be made meaningful for decisions. That is, better data alone are insufficient for extracting meaning. All decisions happen in the context of alternatives, values, and uncertainty. Without such context, scientific accuracy alone fails to guide decision making and action.

Although there might be benefits to promote LCA as a value-free, scientifically objective instrument (not the least of which is to confer the prestige of science upon the analysts), proclaiming that LCA is free from subjective value choices is disingenuous. In fact, LCA is fraught with value-laden choices, including: establishing a goal, selecting a functional unit, considering alternatives, selecting assessment criteria, and choosing the geospatial and temporal resolution brought to bear in estimation of characterization factors. Such value choices do not undermine the credibility of the study, so long as they are documented, defensible, and can be reproduced by others. Thus, efforts to shield these choices behind a veil of scientific objectivity may have the perverse consequence of discrediting, rather than enhancing, the reputation of the analysis.

\section{Supporting Decisions with Life Cycle Assessment}

Without supporting decisions, LCA fails to fulfill its promise as an analytic approach with the power to improve the state of the environment. Whereas consensus standards and methodological codes are an important step in establishing scientific reproducibility, without innovation that challenges and improves those standards the community will risk becoming irrelevant as more useful tools (e.g., footprinting) gain attention. The evidence is now convincing that typical practices in normalization have been unsuccessful, and further efforts to improve the current paradigm of existing reference data sets are well past the point of diminishing returns. Until common LCA practice regularly makes use of updated interpretations of normalization, the results of characterized inventories in a comparative LCA will fail to inform discriminating choices among environmentally relevant alternatives.

\section{References}

Bjorn, A. and M. Z. Hauschild. 2015. Introducing carrying capacitybased normalisation in LCA: Framework and development of references at midpoint level. The International Journal of Life Cycle Assessment 20(7): 1005-1018.

ISO (International Organization for Standardization). 2006. ISO 14044: Environmental management-Life cycle assessmentRequirements and guidelines. Geneva, Switzerland: ISO.

Norris, G. A. 2001. The requirement for congruence in normalization. The International Journal of Life Cycle Assessment 6(2): 85-88.

Pizzol, M., A. Laurent, S. Sala, B. Weidema, F. Verones, and C. Koffler. 2016. Normalisation and weighting in life cycle assessment: Quo vadis? The International Journal of Life Cycle Assessment DOI: 10.1007/s11367-016-119-1.

Prado-Lopez, V., T. P. Seager, M. Chester, L. Laurin, M. Bernardo, and S. Tylock. 2014. Stochastic multi-attribute analysis (SMAA) as an interpretation method for comparative life-cycle assessment (LCA). The International Journal of Life Cycle Assessment 19(2): 405-416.

Conflict of interest statement: The authors have no conflict to declare.

Address correspondence to: Stefano Cucurachi, Bren School of Environmental Science \& Management, University of California, Santa Barbara, Bren School, 2400A, Santa Barbara, CA 93106, USA. Email: scucurachi@bren.ucsb.edu 